\title{
Propagation of Currents on a Humanoid Phantom with Skin-Imitating Sheets Mixed with a Normal Saline Solution to Determine the Effect of Embedded Modules
}

\author{
Shinji Kawakura*, Yoshihiro Nakabo*, Kiyoshi Fujiwara* \\ National Institute of Advanced Industrial Science and Technology (AIST), Department of Information \\ Technology and Human Factors, Robot Innovation Research Center 1-1-1 Umezono, Tsukuba, Ibaraki \\ 305-8560 Japan. \\ * Corresponding author. Tel.: +81-80-9781-1535; email: s.kawakura@aist.go.jp \\ Manuscript submitted June 20, 2017; accepted September 5, 2017. \\ doi: 10.17706/ijbbb.2018.8.1.42-52
}

\begin{abstract}
In recent years, further basic research is required for phantoms with artificial skin and bodily fluid/gelatin mixtures containing normal saline solution (NSS). The phantom used in this study mainly consisted of silicon rubber and had small amounts of other chemical substances. The phantom possessed the stature of an average middle-aged to elderly Japanese man. This humanoid phantom, along with other similar ones, has been used in experiments conducted by the communications industry to measure electromagnetic wave streams and diffusion from common cellular phones. In this work, we measured the propagation and diffusion of alternating currents on and within the phantom over measurement frequencies ranging from $5 \mathrm{~Hz}$ to $1 \mathrm{MHz}$. We also partially wrapped the phantom with skin-imitating gel sheets. Finally, we mixed NSS with the gel sheets, and, by analyzing the data obtained, elucidated the effect of common electric currents from medical machines (e.g., low-frequency therapy equipment) on a common pacemaker and other embedded electrical modules. We suggest practical information and advice for further improvements for the physically challenged and the elderly. Our device holds promise for researchers and developers of phantoms and robots used as virtual physical simulators, which can be combined with existing computational simulation software.
\end{abstract}

Key words: Propagation of alternating currents, artificial rubber phantom, gel sheet, normal saline solution, influence of embedded electrical modules.

\section{Introduction}

In recent years, the medical field and the application of diverse electro medical devices have gradually become integrated, as evident in applications in massage therapy, simple symptomatic therapies, and sports science. Diverse electric stimulations, human body measurements, and social-science-based therapies have also seen developments ([1]-[14]). Both outdoor measurement systems and electrically embedded equipment for various real-time body measurements have evolved year after year, for example, in terms of common body condition factors, pacemaker data, and pulse rate meter output ([14]-[16]). For many years, researchers, healthcare personnel, and engineers who use electrical systems have obtained skin and internal body impedance measurements that allowed them to determine requirements for current sources and voltage measurement circuits. Various system designers who use two or more electrode instruments need to exercise additional caution because measurements for internal or superficial conditions in a real 
body or in a simulated body not containing skin or simulated skin substances may yield results that are different from those that include skin or the simulated substances. In the meantime, scientists are developing analytical methodologies and systems that target both the human body surface, internal organs, fats, and muscles, as measures and countermeasures against sudden electric accidents. Studies in bioelectrical engineering at the medical front and in other common areas has been progressing significantly ([2]-[4], [17]-[26]). Nevertheless, the subjects of these studies vary widely in terms of race, age, career, and other parameters. Experiments in this field of research utilize software-based simulators, non-contact methods, and real human bodies so long as the experimental settings comply with official regulations and public laws ([27]). Until now, the targeted achievements concerning living human bodies and non-living humanoid devices have been limited.

Rigid regulations and difficulties concerning the safety, research ethics, and legal aspects of the above studies persist. That is why researchers and medical service workers cannot attach chemical substances, pierce the human body, or embed electronic modules (pacemakers, electrical stimulation devices, etc.) into the human body for experimentation. In this study, we performed measurements on an artificial, custom-made physical simulator phantom to avoid complications such as risks linked to cutting a real body, inserting electrodes, and changing skin conditions (Fig. 1). Its metallic mold was carefully designed in its early phase of production. The very-high-frequency range (200 MHz or below), the phantom and $0.18 \mathrm{wt} \%$ saline solution are similar with regard to the real part of their inductive capacity $\left(\varepsilon^{\prime}\right)$ and conductance $(\mathrm{S} / \mathrm{m})$. Therefore, the phantom is a suitable physical simulator for this study. However, we did not calculate phase differences because of methodological considerations. Asahi Rubber Inc., Japan, and other research institutes (e.g., Graduate School of Hokkaido University, Sapporo, Japan) have manufactured phantoms rather similar to the phantom we used in this study and have developed them for years in order to provide data related to their electromagnetic characteristics. We used the Red Dot 2330 electrode (3M Health Care, 3M United States, MA)-a standard in the field-for electrocardiography examination, as shown in Fig. 2.

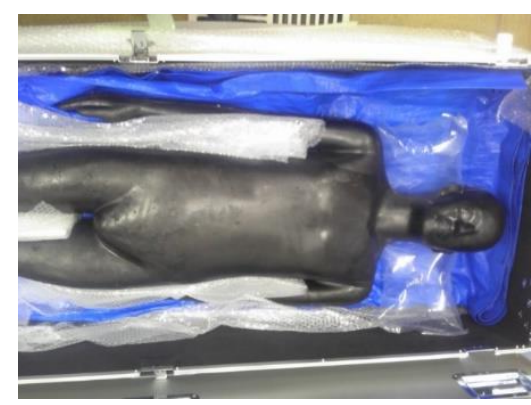

Fig. 1. Default rubber phantom.

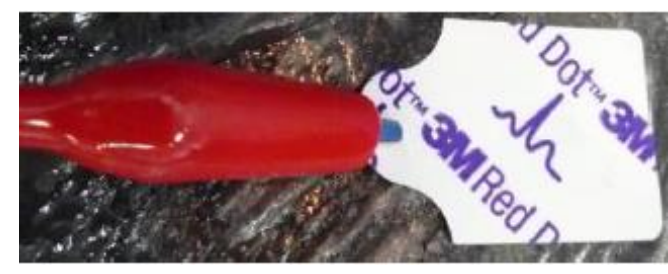

Fig. 2. Red dot 2330 .

Initially, the main aim was to use the phantom for diverse experiments to analyze the propagation and diffraction of radio waves from common cell phones. In the first phase of this study, we measured the rate of change in voltage penetrating through the default phantom. In the second phase, we partially wrapped the phantom's surface with an artificial gel sheet "NR" (Hydro-gel for electrical polar plates; Sekisui Kasei 
Manufacturing Inc., Japan). In the third phase, we filled the vacant spaces between the phantom and these sheets, as well as subtle cracks in the phantom, with normal saline solution (NSS; $0.9 \mathrm{wt} \%$ ) using a brush and spray bottle. In addition, we changed both the shape of the gel sheets and volume according to the settings of electric current measurements. By focusing on both recent and traditional bioelectrical capabilities, as well as related experimental data for the typical human body, researchers have verified the usefulness of the experimental method to some degree. The present study thus constructed and demonstrated a simulation and measurement system for use in an indoor experimental laboratory and in other testing sites. The purpose of our research is to prove the utility of this phantom in terms of the rate of change of the current voltage and its profiles.

\section{Theory and Methodology}

\subsection{Theory}

The intractableness of the measured $\sigma, \varepsilon^{\prime}$, and impedance of various body parts has been heavily debated for many years ([2]-[4], [21]). Researchers have analyzed the ratio of segmental impedance values. The impedance of muscles and organs can reach high levels. In general, skin, muscles, organs, bones, and circulating blood of subjects have diverse characteristics, which is why simulating the human body is inherently complicated. Researchers have thus had great difficulty in discussing the electronic complexity of measurements on non-living objects. We investigated the current-carrying characteristics of this phantom and then demonstrated the validity of the methods, which are relevant to bioelectric measurements in electrical safety technology.

The height of the phantom was $165.0 \mathrm{~cm}$, which corresponds to the average stature of a middle-aged to old Japanese man (165.9 cm, 60-69 years old; [28]). Furthermore, the size of each phantom part was almost identical to that of the corresponding part of the average-stature body. The angles of both infra-axillary sites and crotches were set at 30 degrees. Manufacturers set those angles as a standard for the subject form so that the phantoms can be used in basic studies in medical institutions or medical research institutions.

The relative resistance of the NR gel sheet for direct current (DC) was $2 \times 10^{4} \Omega \cdot \mathrm{cm}$ on one side, while the impedance on the other was $100 \Omega$. Preliminary measurements on the gel revealed that these values could change according to the measurement frequencies. The thickness of the gel sheet was around $1.0 \mathrm{~mm}$. There are many previous studies on the electrical resistances and impedances of human skin ([2]-[4], [16], [20], [21]). In general, the resistance of human skin to a DC source is around $10^{4} \Omega \cdot \mathrm{cm}$ under dry conditions, dropping to $800 \Omega \cdot \mathrm{cm}$ under wet conditions. The thickness of the epidermis ranges from 0.06 to $0.2 \mathrm{~mm}$, whereas that of the dermis ranges from $2.0 \mathrm{~mm}$ to $2.2 \mathrm{~mm}$. Taking these into consideration, we deemed the gel sheet to be the most appropriate option as far as commercial availability and utility in laboratory experiments were concerned.

From an electrical engineering standpoint, other researchers have noted that the skin impedance of low-frequency bands occasionally reaches extremely high values. The difficulty of incorporating variations in the impedance values into the reconstruction algorithm leads to large distortions ([2]-[4], [29]). These have thus motivated researchers, healthcare personnel, and engineers of bio-potential systems to achieve high input impedances over the frequency range of measurements in order to prevent an imbalance in skin impedance that would otherwise convert common-mode voltage to differential voltage. This study specifically focused on the heart and examined the electrode(s) of embedded electrical modules attached to a patient's heart, as in common pacemakers. The human and animal heart is a muscular organ that pumps blood through blood vessels. The human heart is roughly at the center of the breast (mediastinum), slightly extending leftward behind the breastbone at the level of the thoracic vertebrae T5 to T8. The mass of an adult heart varies between 250 to $350 \mathrm{~g}$, and its size is comparable to that of the fist. 
Common artificial cardiac pacemakers for the Japanese market are typically embedded near the middle of the left collarbone or on the right/left upper side of the heart, with the electrical lead line(s) extending to the user's heart. Its diameter is between 40 to $50 \mathrm{~mm}$, its thickness is around 5-6 mm, and it weighs approximately 12 to $30 \mathrm{~g}$ (e.g., Sure Scan, Medtronic Inc., Japan). We investigated the electrical specifications of pacemakers by examining one of the most dominant modules, the name of which is withheld for privacy reasons. The constant output voltage was $0.1-10.0 \mathrm{~V}$ (5.0 V average), and the constant electric current was 0.1-20.0 mA (10.0 mA average). The pulse width was $0.3-2.0 \mathrm{~ms}$ and the sensitivity was 1.0-5.0 mV. We did not define the frequency, however, since it is dependent on the intended usage.

The leading embedded electronic modules used for sacral nerve stimulation, such as the InterStim Model 3023 and InterStim II Model 3058 (Medtronic Inc., Japan), have dimensions of $44 \mathrm{~mm} \times 51 \mathrm{~mm} \times 8 \mathrm{~mm}$. Operators can set them near the upper end of the pelvis to stimulate the third sacral nerve. In this case, the constant output voltage is $0.0-10.5 \mathrm{~V}$, the pulse width is $180-240 \mu \mathrm{s}$, and the frequency is $10.0-14.0 \mathrm{~Hz}$. In addition, we reviewed popular low-frequency medical devices in Japan that send signals at several kilohertz from the user's waist. For example, the "Ene-pulse" sends a $\sim 4000 \mathrm{~Hz}$ alternating-current signal, which is similar to that of other electronics. Therefore, the data presented in this study, especially those within the 5, $10,20,10^{3}$, and $10^{4} \mathrm{~Hz}$ range, are a significant and useful reference for the aforementioned research areas. Our data may provide insight for other researchers for future phantom and robot developments.

\subsection{Methodology}

Experiments using a phantom, peripheral substances, and measurement devices were performed in this study to address highly complex electronic specifications and features. We present detailed results on changes in voltage magnitudes and trends with respect to gel sheet size, measurement points, and frequencies. We investigated three superficial settings: the default phantom (as described previously), the phantom wrapped with a gel sheet, and the phantom wrapped in a gel sheet with an NSS pattern, which replicates a real human body with elastic skin.

In the first phase of this study, we performed measurements on the default phantom body. In the second phase, we partially wrapped the phantom with an artificial NR gel sheet and repeated the measurements. Finally, in the third phase, the authors filled the minute vacant spaces between the phantom and the NR gel sheets, as well as the subtle cracks in the phantom, with NSS using a brush and spray bottle. The specifications of the function generator, electrodes, and gel sheets used in this study are described here. The function generator, a 33522A-2-Channel $30 \mathrm{MHz}$ function (arbitrary waveform) generator (Keysight Technologies Inc., Japan), was used on various experimental sites. We used currents having sinusoidal waves at frequencies ranging from $5 \mathrm{~Hz}$ to $1 \mathrm{MHz}$ for the measurements. Colominas et al. [21] used frequencies of $1,10,10^{2}, 10^{3}, 10^{4}, 10^{5}$, and $1 \mathrm{MHz}$ to measure human skin impedance. Geddes et al. [30] used frequencies of $10,10^{2}, 10^{3}$, and $10^{4} \mathrm{~Hz}$ to measure human lung, muscle, and blood impedance. We utilized Red Dot 2330 electrode patches (3M Health Care, 3M United States, MA) to conduct electrocardiography examination. The patches were $0.5 \mathrm{~mm}$ thick and provided a skin contact area of 20 $\mathrm{mm} \times 20 \mathrm{~mm}$. Previous work has validated the experimental procedure and its peripheral systems.

Fig. 3-5 present the detailed configuration of the experimental setup. We connected the positive electrode of the function generator to the right side of the phantom, and the negative electrode to its left. Similarly, we attached the positive needle-shaped electrode of the oscilloscope to the right side of the phantom and the negative electrode to its left. Subsequent measurements were performed by varying the location of the electrode patches ([2]-[4], [21], [31]). In this study, however, we only present data for measurement points of the vertical-line site located at the heart, which is $380 \mathrm{~mm}$ from the function generator's two parallel electrodes with $180 \mathrm{~mm}$ center-to-center spacing. 


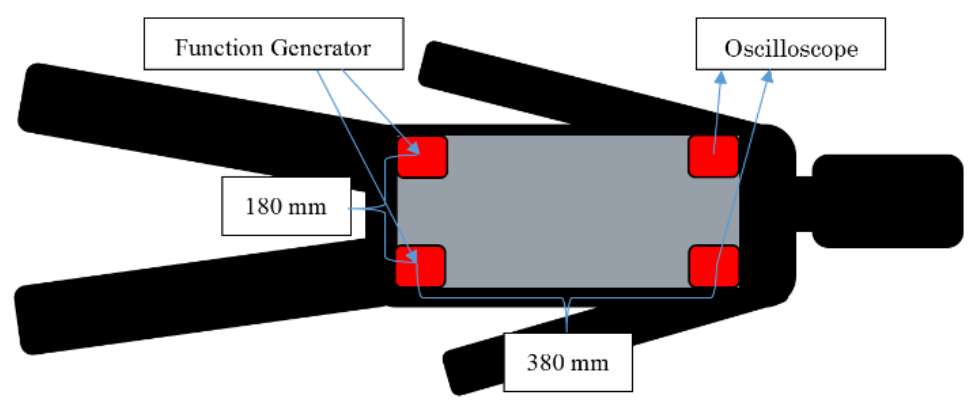

Fig. 3. Schematic of measurements on the phantom with one $200 \mathrm{~mm} \times 500 \mathrm{~mm}$ rectangular sheet under the electrode patches of both the function generator and oscilloscope.

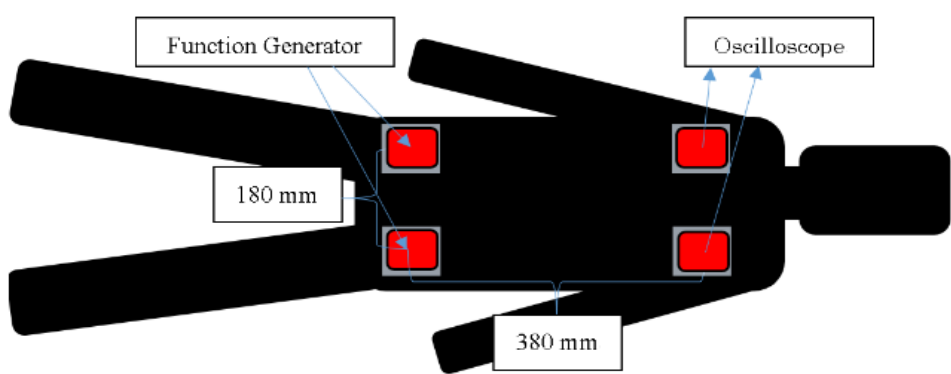

Fig. 4. Schematic of measurements on the phantom with four $50 \mathrm{~mm} \times 50 \mathrm{~mm}$ square sheets under the electrode patches of both the function generator and oscilloscope.

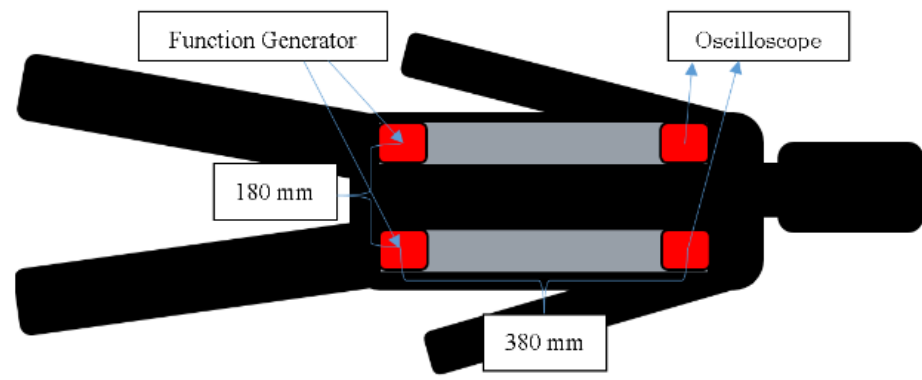

Fig. 5. Schematic of measurements on the phantom with two $50 \mathrm{~mm} \times 500 \mathrm{~mm}$ rectangular sheets under the electrode patches of both the function generator and oscilloscope.

We also varied the size of gel sheets that were cut and attached to the front side of the phantom's trunk. The dimensions of the patterns were $200 \mathrm{~mm} \times 500 \mathrm{~mm}, 50 \mathrm{~mm} \times 50 \mathrm{~mm}$, and $50 \mathrm{~mm} \times 500 \mathrm{~mm}$ (Fig. 3-5). We then calculated the average rate of change in the measurement current voltage on the basis of two or three replicates per configuration for each span from $5 \mathrm{~Hz}$ to $1 \mathrm{MHz}$.

\section{Results}

Fig. 6-8 depict the differences in the measurement current voltage for the aforementioned setups, which emit current voltages. We calculated the average rate of change of the measurement current voltage based on two or three replicates for each experimental setting. We used measurement frequencies of $5,10,10^{2}$, $10^{3}, 10^{4}, 10^{5}$, and $1 \mathrm{MHz}$ to illustrate the frequency dependence. Fig. 6-8 present the results obtained by varying the experimental settings and by changing the emitted measurement current frequencies and voltages.

The numerical ratios in the graph of Fig. 6-8 corresponding to 1000 and $2000 \mathrm{mVpp}$ are lower than those of 10 or $100 \mathrm{mVpp}$ probably because, in the former two voltages, penetration through the phantom and the gel sheet occurred, resulting in more than half of their ratios dipping below 100 percent. As for data 
obtained at a measurement current under $100 \mathrm{~Hz}$, which are considered to be low frequencies in this area of research, the consequent low penetrability resulted in irregular rates of change. The rate curves over 100 $\mathrm{Hz}$, on the hand, have gentler slopes. A resonance phenomenon around $100 \mathrm{~Hz}$ may be observed in the graph of Fig. 7; the rate curve surpasses the 200 percent mark. On the other hand, a significant resonance phenomenon is apparent at around $10^{3} \mathrm{~Hz}$ in the graph of Fig. 8. Mixing NSS could shift the peak points of the phenomenon, which we believe to be an important consideration in constructing anatomical models of the human body.

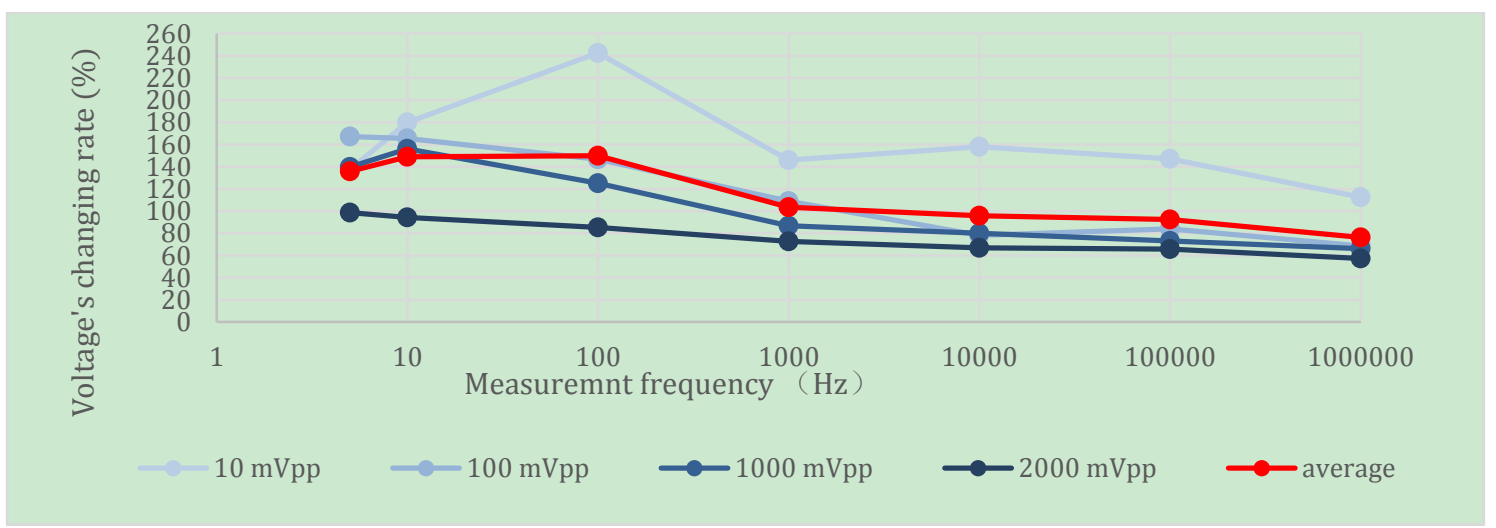

Fig. 6. The rate of change in voltage on the site located at the heart of the default phantom with varying emitted current voltage.

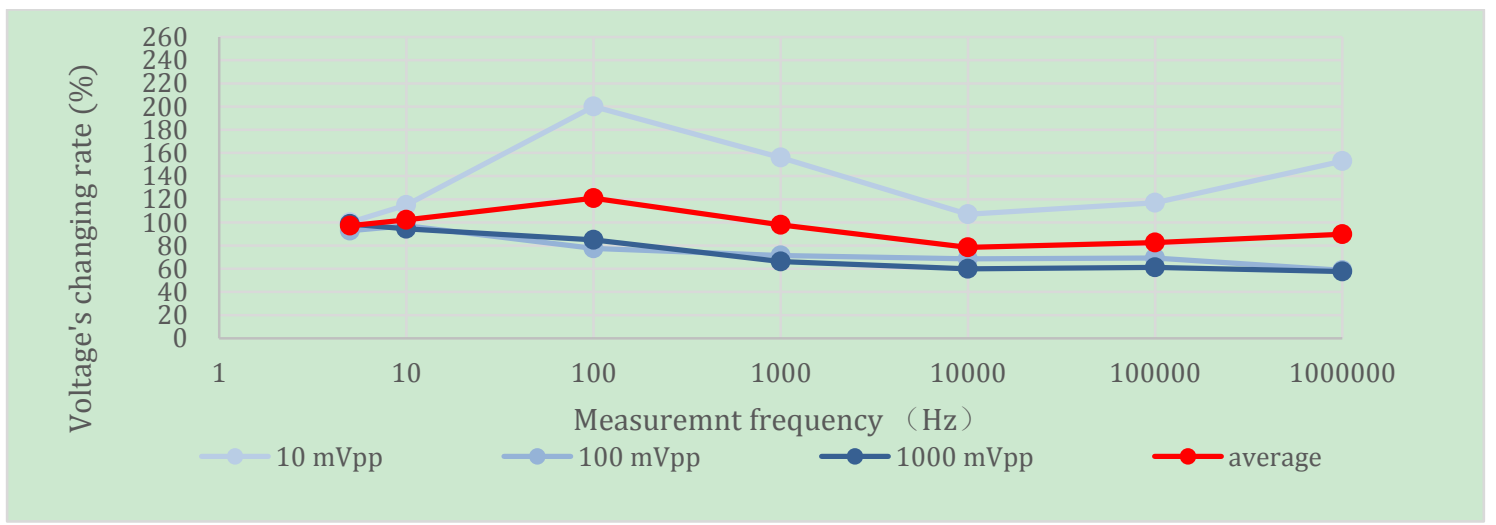

Fig. 7. The rate of change in voltage on the site located at the heart of the e phantom wrapped with an NR gel sheet $(200 \mathrm{~mm} \times 500 \mathrm{~mm})$ with varying emitted current voltage.

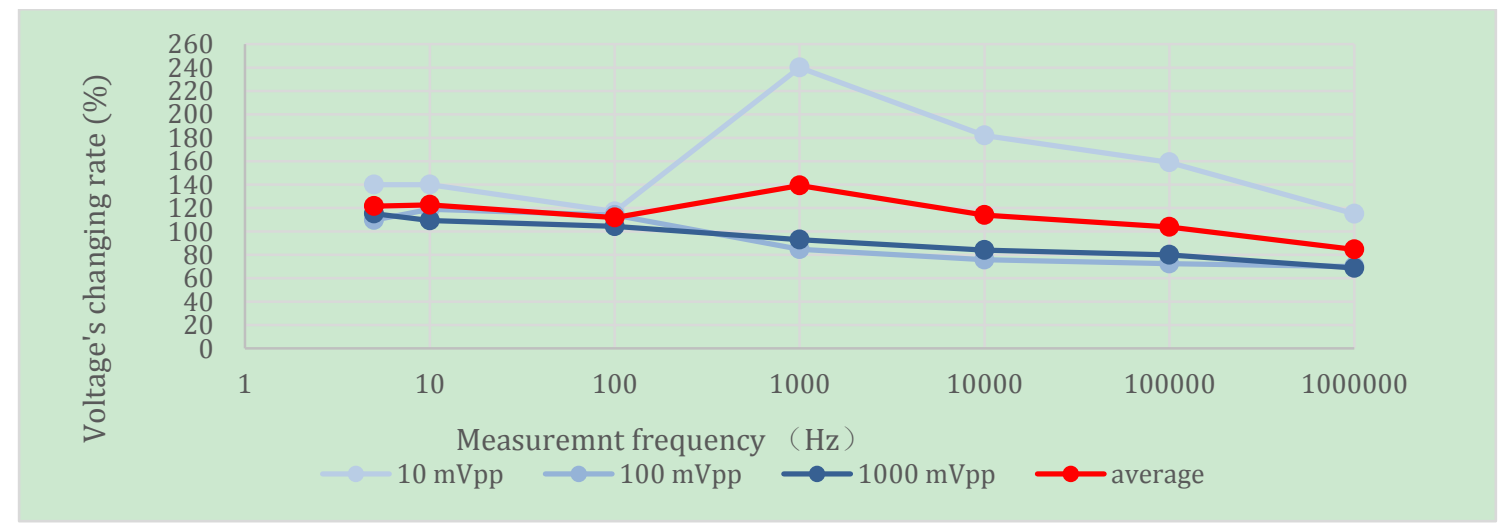

Fig. 8. The rate of change in voltage on the site located at the heart of the phantom wrapped with the same sheet $(200 \mathrm{~mm} \times 500 \mathrm{~mm})$ and with NSS with varying emitted current voltage. 
Fig. 9-11 show the calculated average rate of change in the measurement current voltage, in ohms, based on two or three replicates per configuration for each span from $5 \mathrm{~Hz}$ to $1 \mathrm{MHz}\left(5,10,20,50,10^{2}, 10^{3}, 10^{4}\right.$, $10^{5}, 5 \times 10^{5}$, and $1 \mathrm{MHz}$ ). As a whole, the numerical ratios decrease with increasing measurement current voltage. It is difficult, however, to account for the trends observed within the $5-10^{4} \mathrm{~Hz}$ range in the graph of Fig. 11. We attribute the irregular fluctuations of the rate curves to resonance phenomena. The rate curves at frequencies over $10^{3} \mathrm{~Hz}$ in the graph of Fig. 10 and 11 are likely to be under 100 percent. In particular, the configurations with the NR gel and NR gel with NSS tended to settle to 100 percent, especially at frequencies below $10^{3} \mathrm{~Hz}$. This result indicates that the superficial gel and NSS selectively accepted both the measurement and penetrating currents.

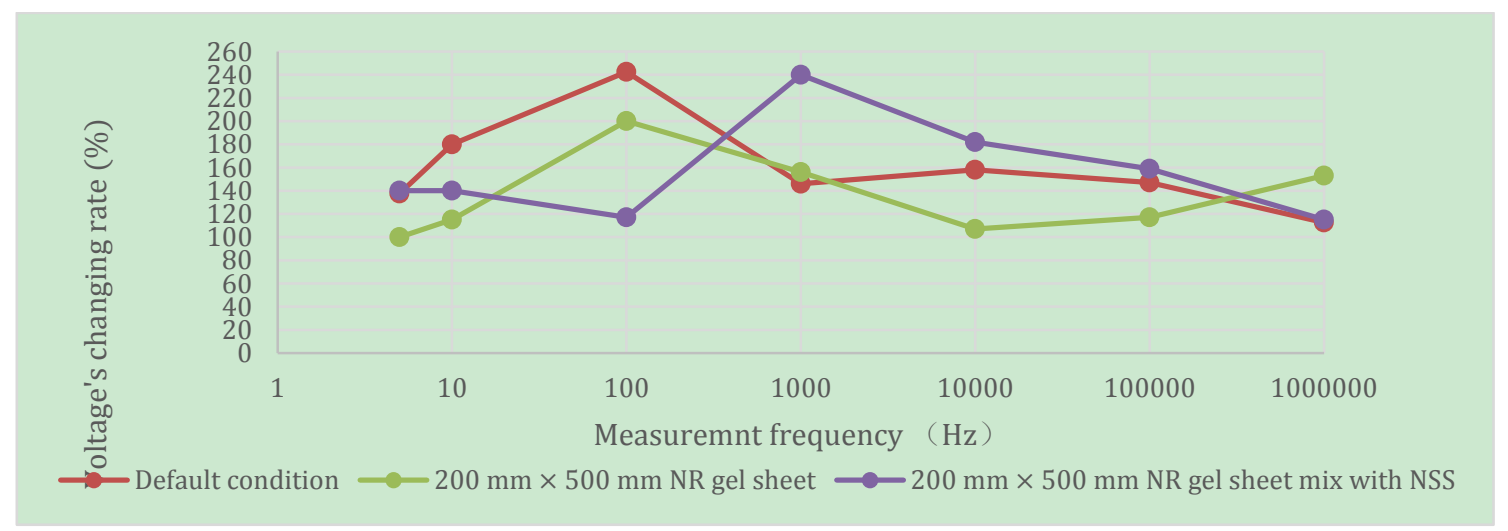

Fig. 9. The rate of change in voltage on the site located at the heart of the phantom with varying emitted current frequencies at $10 \mathrm{~mA}$.

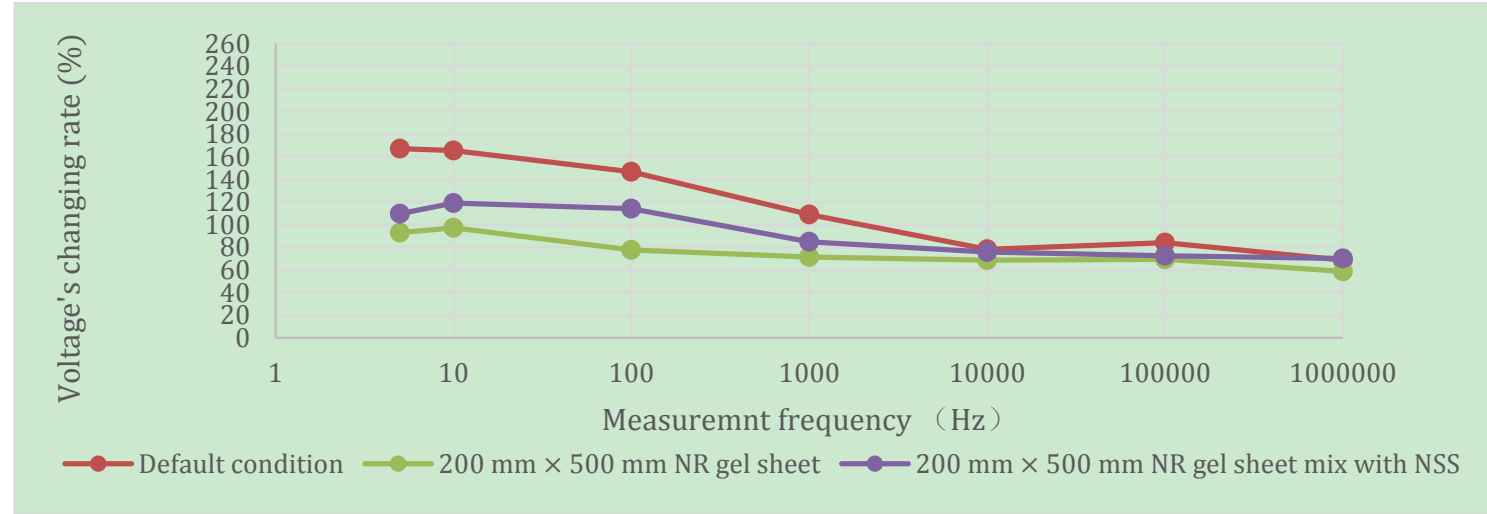

Fig. 10. The rate of change in voltage on the site located at the heart of the phantom with varying emitted current frequencies at $100 \mathrm{~mA}$.

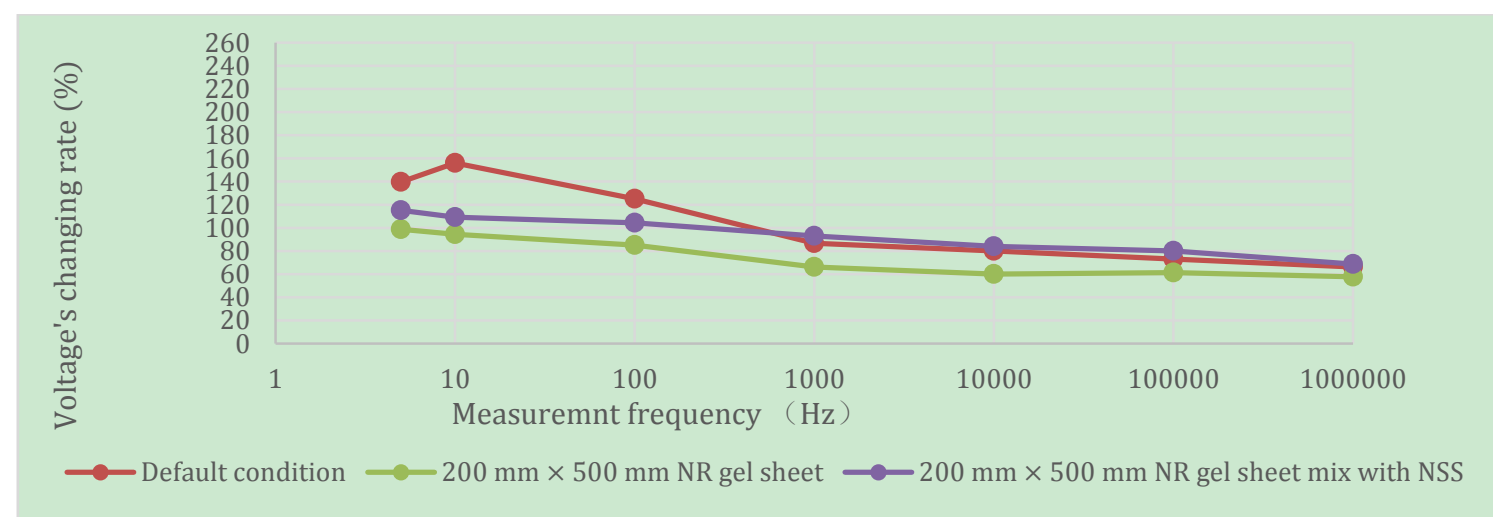

Fig. 11. The rate of change in voltage on the site located at the heart of the phantom with varying emitted current frequencies at $1000 \mathrm{~mA}$. 
We changed the size of the NR gel sheets that were cut and attached to the front side of the phantom's trunk. Three configurations were used: one $200 \mathrm{~mm} \times 500 \mathrm{~mm}$ rectangular sheets under the four corners of the electrode patches (Fig. 3), four $50 \mathrm{~mm} \times 50 \mathrm{~mm}$ square sheets under the electrode patches of the function generator and oscilloscope (Fig. 4), and two $50 \mathrm{~mm} \times 500 \mathrm{~mm}$ rectangular sheets that connect the aforementioned points on the electrode patches (Fig. 5), as described previously. We did not include NSS in these cases.

Fig. 12 displays the successive average rates of change in voltage measured on the site located at the heart, in ohms, for each span from $5 \mathrm{~Hz}$ to $1 \mathrm{MHz}\left(5,10,20,50,10^{2}, 10^{3}, 10^{4}, 10^{5}, 5 \times 10^{5}\right.$, and $\left.1 \mathrm{MHz}\right)$. Some data are lacking for methodological reasons. The three data lines are tightly clustered together at frequencies higher than $10^{4} \mathrm{~Hz}$. Below the $10^{4} \mathrm{~Hz}$ range, however, the area of the $50 \mathrm{~mm} \times 500 \mathrm{~mm}$ and 200 $\mathrm{mm} \times 500 \mathrm{~mm}$ NR gel sheets probably maintained the voltage rate of change relative to that of the default phantom. These results imply that the measurement currents run selectively near the phantom surface; in practical terms, they imply that developers and researchers have to carefully inspect its area and material when using the artificial skin.

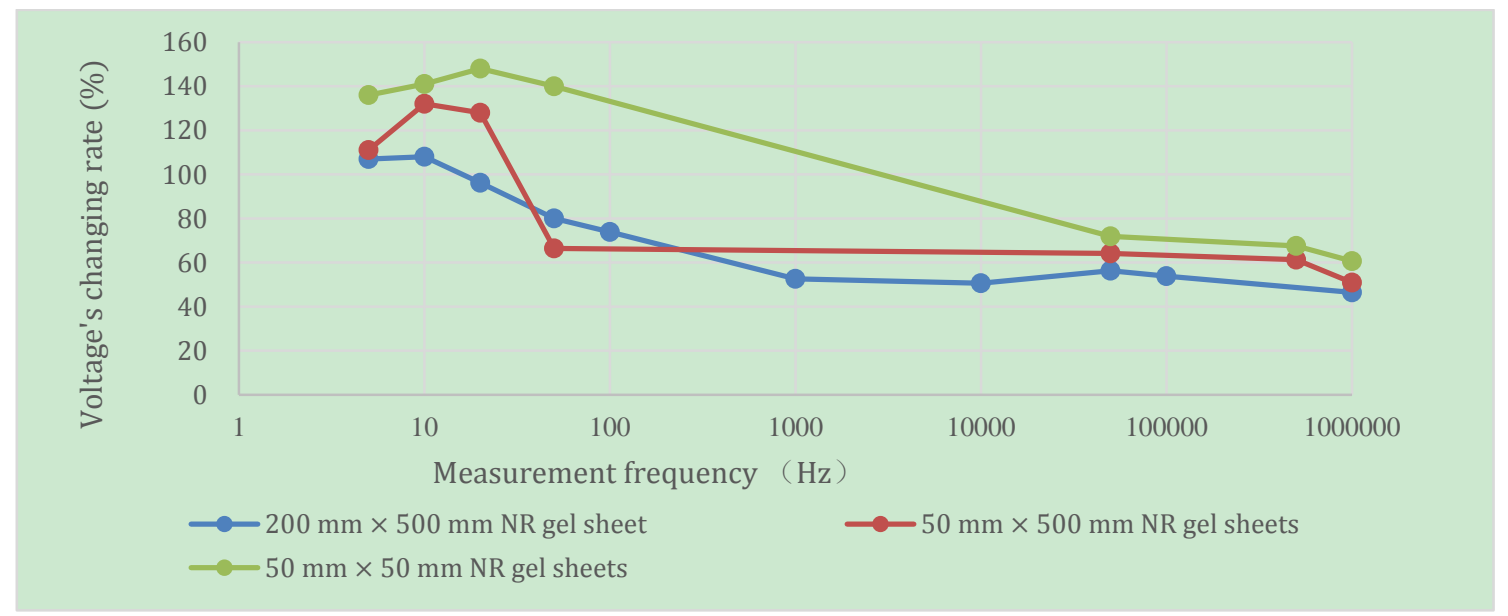

Fig. 12. The rate of change in voltage on the site located at the heart of the phantom wrapped with rectangular NR gel sheets according to three configurations.

\section{Discussion and Conclusion}

After selecting the phantom, gel sheet, NSS, and measurement methodologies, we estimated the rate of change in the measurement current voltage amplitude on and into the phantom. Our results demonstrate a promising physical simulator with diverse potential uses at research institutes, academic institutions, and hospitals. We believe that combined with experimentation, these results may assist researchers and healthcare workers in overcoming difficulties in measurements on phantoms and robots more comprehensively. Additionally, we confirmed and reconsidered various measurement and performance limitations, especially regarding currents from a standard function generator. Overall, we obtained meaningful results and consequent benefits from this study, which specifically concerns the phantoms used with chemical substances, NSS and peripheral devices. Therefore, we encourage the use of chemicals in fabricating similar electromagnetic dolls or robots.

We believe that all our data for the phantom and gel sheet slightly differ from those in other studies ([32]-[36]) because of the presence of internal cracks of different sizes. In addition, insufficiencies and imbalances in compositions (mainly in terms of silicon rubber, specific chemical substances, and NSS) forced us to reselect the compositions for the measurement frequencies. We will determine the validity, 
durability, precision, long-term effects, and above all, the social impact, of this system in future work. In subsequent studies, we aim to obtain a greater variety of electrical data and to include studies on phantoms modeled after other races and after infants in order to facilitate comparisons with real human body parts and tissues. Other methodologies, such as those using other timelines of trials, tools, specific conditions of subjects, and experimental spaces, should be also investigated in future studies. Regardless of the path that we will follow and the human models we will create, we have to logically deliberate the diverse problems related to measurement frequencies.

The proposed systems have future prospects in cutting-edge bioelectronics and bionomy that contribute to improved internal anthropometry. We may measure the phantom's electromagnetic properties (i.e., power distribution and current-carrying property), as well as its imaginary organs, fat, and muscle volumes by bioelectrical impedance measurement and analysis. One of the most widely accepted theories is the bio impedance analysis theory, from which various methodologies have been evolving. The data presented here may be useful for the fabrication of phantoms, dolls, and robots. For instance, the developers of all Physion series theories have annotated and used the BI (Bio- electrical Impedance) method for the analysis of human muscle volume. Finally, our data indicate a promising outlook for developing practical supports for physically challenged and elderly people.

\section{Acknowledgment}

The author's heartfelt appreciation goes to colleagues at National Institute of Advanced Industrial Science and Technology (AIST), members of Impulsing Paradigm Change through Disruptive Technologies (ImPACT) Program / Cabinet Office, Prof. Yoshiyuki SANKAI, Government of Japan, researchers at the National Institute of Occupational Safety and Health (JNIOSH), Prof. Yoshihisa MASUO at Muscle Lab Inc., Associate Prof. Megumi OHTA at Chukyo University, Yatoro Electronics Co., Ltd., NIPPON SHOOTER Ltd., Medical Task Force Co., Ltd., who have provided careful supports, feedbacks and valuable comments.

\section{References}

[1] Thomasset, M. A. (1962). Bioelectric properties of tissue. Impedance measurement in clinical medicine. Significance of Curves Obtained, Lyon Medical, 94, 107-118.

[2] Gabriel, C., Gabriel S., Corthout, E. (1996). The dielectric properties of biological tissues I. Literature survey. Physics in Medicine and Biology, 41(11), 2231-2249.

[3] Gabriel, C. (1996). The dielectric properties of biological tissue II: Measurements in the frequency range $10 \mathrm{~Hz}$ to $20 \mathrm{GHz}$. Physics in Medicine and Biology, 41(11), 2251-2269.

[4] Gabriel, C., Gabriel, S., \& Lau, R. (1996). The dielectric properties of biological tissues: III. Parametric models for the dielectric spectrum of tissues. Physics in Medicine and Biology, 41(11), 2271-2293.

[5] Miyatani, M., Kanehisa, H., \& Fukunaga, T. (2000). Validity of bioelectrical impedance and ultrasonographic methods for estimating the muscle volume of the upper arm. European journal of applied physiology, 82, 391-396.

[6] Miyatani, M., Kanehisa, H., Masuo, Y., Ito, M., \& Fukunaga, T. (2001). Validity of estimating limb muscle volume by bioelectrical impedance. Journal of applied physiology, 91(1), 386-394.

[7] Miyatani, M., Kanehisa, H., Ito, M., Kawakami, Y., \& Fukunaga, T. (2004). The accuracy of volume estimates using ultrasound muscle thickness measurements in different muscle groups. European Journal of Applied Physiology, 91, 264-272.

[8] Ishiguro, N., Kanehisa, H., Miyatani, M., Masuo, Y., \& Fukunaga, T. (2005). A comparison of three bioelectrical impedance analyses for predicting lean body mass in a population with a large deference in muscularity. Eur. J. Appl. Physiol., 94, 25-35. 
[9] Ishiguro, N., Kanehisa, H., Miyatani, M., Masuo, Y., \& Fukunaga, T. (2005). Applicability of segmental bioelectrical impedance analysis for predicting trunk skeletal muscle volume. Journal of Applied Physiology, 100(2), 572-578.

[10] Miura, K., Kawagoe, M., \& Koh, S. (2007). Characteristics of functional status among care requiring community-dwelling frail elderly. Journal of Human Life Science, 6, 1-10.

[11] Tanaka, N. I., Miyatani, M., Masuo, Y., Fukunaga T., \& Kanehisa, H. (2007). Applicability of a segmental bioelectrical impedance analysis for predicting the whole body skeletal muscle volume. J. Appl. Physiol., 103, 1688-1695.

[12] Yonei, Y., Miwa, Y., Hibino, S., Takahashi, Y., Miyazaki, R., Yoshikawa, T., Moriwaki, H., Hasegawa, T., Hiraishi, T., \& Torii, K. (2008). Japanese anthropometric reference data special emphasis on bioelectrical impedance analysis of muscle mass. Anti-Aging Medicine, 5(6), 63-72.

[13] Nomoto, K., Miyazaki, R., \& Hasegawa, T. (2010). Efficacy of a health promotion program with anti-aging medical checkup and instructions for walking under pedometer management in factory workers. Anti-Aging Medicine, 7, 73-84.

[14] Suzuki, I. (2011). Study of characteristic and the faculty decline item of the activities of daily living actual situation of nursing care preventive-service user. Medical and Health Science Research: Bulletin of Tsukuba International University, 2, 167-174.

[15] Miyazaki, R., Hasegawa, T., \& Fujioka, N. (2009). Effects on anti-aging indicators in middle-aged men of an intervention to prevent lifestyle-related diseases: Pilot study utilizing a companywide anti-aging medical checkup and pedometers. Anti-aging Medicine, 6, 83-94.

[16] Ichikawa, N., \& Tomita, H. (2009). Basic Facts about Electric Shocks and Fatal Accident Statistics for the Last 30 Years (Report No. 25). Tokyo: The national institute of occupational safety and health.

[17] Nyboer, J. (1959). Electrical impedance plethysmography. Illinois: Charles C Thomas Publisher. Quarterly Journal of Experimental Physiology and Cognate Medical Sciences, 45(3).

[18] Hoffer, E. C., Meador, C. K., \& Simpson, D. C. (1969). Correlation of whole-body impedance with total body water volume. Journal of Applied Physiology, 27(4), 531-534.

[19] Namie, M., Haeno, M., Sakamoto, K., Kanai, H., \& Tagawa, H. (1985). Estimation of fluid distribution by impedance method. Japanese Society for Medical and Biological Engineering, 23(6), 354-360.

[20] Kim, Y., \& Woo, W. H. (1987). A prototype system and reconstruction algorithms for electrical impedance technique in medical body imaging. Clin. Phys. Physiol. Meas., 8, suppl., A, 63-70.

[21] Colominas, J., Rosell, J., Webster, G. J., Rosell, J., Riu, P., \& Pallas-Areny, R. (1988). Skin impedance from 1 $\mathrm{Hz}$ to $1 \mathrm{MHz}$. IEEE Transactions on Biomedical Engineering, 35(8), 649-651.

[22] Brown, B. H., Karatzas, T., Nakielny, R., \& Clarke, R .G. (1988). Determination of upper arm muscle and fat areas using electrical impedance measuments. Clinical physics and physiological measurement, 9(1), 47-55.

[23] Baumgartner, R. N., Chumlea, W. C., \& Roche, A. F. (1990). Bioelectric impedance for body composition. Exercise and Sport Sciences Reviews, 18, 193-224.

[24] Baumgartner, R. N., Ross, R., \& Heymsfield, S. B. (1998). Does adipose tissue influence bioelectric impedance in obese men and women? Journal of Applied Physiology, 84(1), 257-262.

[25] Nakamura, T., Yamamoto, Y., Yamamoto, T., \& Tsuji, H. (1992). Fundamental characteristics of human limb electrical impedance for biodynamic analysis. Medical and Biological Engineering and Computing, $30(5), 465-472$.

[26] Foster, K. R., \& Lukaski, H. C. (1996). Whole-body impedance--what does it measure? The American 86 Journal of Clinical Nutrition, 64, 388-396.

[27] Abe, T., Kondo, M., Kawakami, Y., \& Fukunaga, T. (1994). Prediction equations for body composition of 
Japanese adults by B-mode ultrasound. American journal of human biology, 6, 161-170.

[28] Ministry of Health, Labor and Welfare, Japan. (2013). Public Welfare Statistics Handbook, 2(6).

[29] Gisser, D. G., Isaacson, D., \& Newell, J. C. (1987). Current topics in impedance imaging. Clin. Phys. Physiol. Meas. 8, Suppl. A., BME-34, 39-46.

[30] Geddes, L. A., et al. (1967). The specific resistance of biologicalmaterials. Med. \& Biol. Engng., 5, 271.

[31] Kubicek, W. G., et al. (1966). Development and evaluation of an impedance cardiographic system to measure cardiac output. Aerospace Med., 37, 1208.

[32] Inoue, M., Taito, K., Takahashi, M., \& Ito, K. (2014). Development of coagulation device for biological tissue using microwave energy. The IEICE Transactions on Electronics, J97-C., 5, 218-224.

[33] Koshiji, F., \& Sasaki, K. (2009). Impedance matching and electrode structure design for wearable transmitter in intra-body transmission. Japan Institute of Electronics Packaging (JIEP), 12(3), 221-232.

[34] Osswald, H. F., Asper, R., Dimai W., \& Simon, W. (1979). On-line continuous potentiometric measurement of potassium concentration in whole blood during open-heart surgery. Clin. Chem., 25-39.

[35] Shitara, M. (2006) Continuing electric stimulation with acupuncture for low back pain. J. Lumbar Spine Disord., 12(1), 143-148.

[36] Suga, K., \& Sasaki, K. (2003). Efficiency of electrical and magnetic stimulation for treatment of urinary incontinence - Using computer model of female abdomen. Japanese Society for Medical and Biological Engineering (JSMBE), 41(3), 213-220.

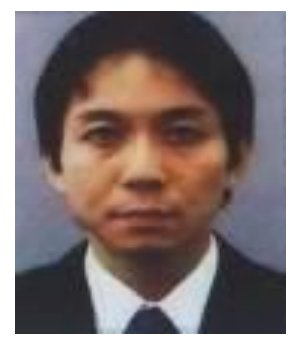

Shinji Kawakura works in research associate at National Institute of Advanced Industrial Science and Technology (AIST)/Department of Information Technology and Human Factors. He received the Ph.D. in environmentology, the University of Tokyo, 2015. He got the B.A in control system engineering, Tokyo Institute of Technology, 2003; the M.A. in human-factors engineering, Tokyo Institute of Technology, 2005. (Meguro-ku, Tokyo, Japan).

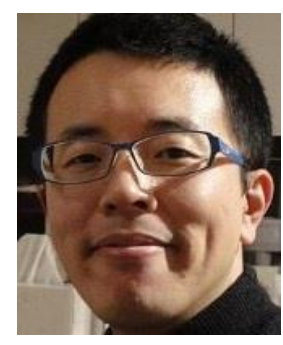

Yoshihiro Nakabo is the team leader at National Institute of Advanced Industrial Science and Technology (AIST)/Department of Information Technology and Human Factors. He received the Ph.D. in engineering, the University of Tokyo, 2000.

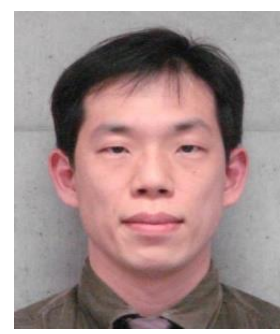

Kiyoshi Fujiwara is senior researcher at National Institute of Advanced Industrial Science and Technology (AIST)/Department of Information Technology and Human Factors. He received the Ph.D. in engineering, Tsukuba University. 